\title{
$\mathrm{Na}_{3} \mathrm{Ti}_{3} \mathrm{O}_{3}\left(\mathrm{SeO}_{3}\right)_{4} \mathrm{~F}$ : A Phase Matchable Nonlinear Optical Crystal with Enlarged SHG Intensity and Band Gap
}

Sheng-Nan Yana,b, ${ }^{\mathrm{a}}$, Xiao-Xue Wang ${ }^{\mathrm{a}, \dagger}$, Chun-Li Hu*a, Bing-Xuan Li ${ }^{\mathrm{a}}$, Fang Kong ${ }^{*}, \mathrm{a}, \mathrm{c}$, Jiang$\mathrm{Gao} \mathrm{Mao}^{\mathrm{a}}$

${ }^{a}$ State Key Laboratory of Structural Chemistry, Fujian Institute of Research on the Structure of Matter, Chinese Academy of Sciences, Fuzhou 350002, P. R. China.

${ }^{b}$ College of Chemistry, Fuzhou University, Fuzhou 350108, P. R. China.

'University of Chinese Academy of Sciences, Beijing 100039, P. R. China

*e-mail: kongfang@fjirsm.ac.cn; clhu@fjirsm.ac.cn 


\begin{tabular}{|c|c|c|}
\hline Section & Caption & Page \\
\hline Figure S1 & $\begin{array}{l}\text { The } 3 \mathrm{D} \text { framework formed by } \mathrm{TiO}_{6} \text { octahedral chains and } \mathrm{Se}(1) \mathrm{O}_{3} \\
\text { groups. }\end{array}$ & S3 \\
\hline Figure S2 & $\begin{array}{l}\text { Simulated and experimental XRD powder patterns of } \\
\mathrm{Na}_{3} \mathrm{Ti}_{3} \mathrm{O}_{3}\left(\mathrm{SeO}_{3}\right)_{4} \mathrm{~F} \text { and } \mathrm{Ag}_{3} \mathrm{Ti}_{3} \mathrm{O}_{3}\left(\mathrm{SeO}_{3}\right)_{4} \mathrm{~F} \text {. }\end{array}$ & S4 \\
\hline Figure S3 & $\begin{array}{l}\text { The assignment of XRD powder peaks of } \mathrm{Na}_{3} \mathrm{Ti}_{3} \mathrm{O}_{3}\left(\mathrm{SeO}_{3}\right)_{4} \mathrm{~F} \text { and } \\
\mathrm{Ag}_{3} \mathrm{Ti}_{3} \mathrm{O}_{3}\left(\mathrm{SeO}_{3}\right)_{4} \mathrm{~F} \text { after TGA. }\end{array}$ & S5 \\
\hline Table S1 & $\begin{array}{l}\text { Atomic coordinates }\left(\times 10^{4}\right) \text { and equivalent isotropic displacement } \\
\text { parameters }\left(\AA^{2} \times 10^{3}\right) \text { for } \mathrm{Na}_{3} \mathrm{Ti}_{3} \mathrm{O}_{3}\left(\mathrm{SeO}_{3}\right)_{4} \mathrm{~F} \text { and } \mathrm{Ag}_{3} \mathrm{Ti}_{3} \mathrm{O}_{3}\left(\mathrm{SeO}_{3}\right)_{4} \mathrm{~F} \text {. }\end{array}$ & S6 \\
\hline Table S2 & $\begin{array}{l}\text { The bond lengths }(\AA) \text { and calculated bond valences for } \\
\mathrm{Na}_{3} \mathrm{Ti}_{3} \mathrm{O}_{3}\left(\mathrm{SeO}_{3}\right)_{4} \mathrm{~F} \text { and } \mathrm{Ag}_{3} \mathrm{Ti}_{3} \mathrm{O}_{3}\left(\mathrm{SeO}_{3}\right)_{4} \mathrm{~F} \text {. }\end{array}$ & S7 \\
\hline Table S3 & The bond angles (deg.) for $\mathrm{Na}_{3} \mathrm{Ti}_{3} \mathrm{O}_{3}\left(\mathrm{SeO}_{3}\right)_{4} \mathrm{~F}$ and $\mathrm{Ag}_{3} \mathrm{Ti}_{3} \mathrm{O}_{3}\left(\mathrm{SeO}_{3}\right)_{4} \mathrm{~F}$. & S9 \\
\hline Table S4 & $\begin{array}{l}\text { EDS analysis results of } \mathrm{Na}_{3} \mathrm{Ti}_{3} \mathrm{O}_{3}\left(\mathrm{SeO}_{3}\right)_{4} \mathrm{~F} \text { and } \mathrm{Ag}_{3} \mathrm{Ti}_{3} \mathrm{O}_{3}\left(\mathrm{SeO}_{3}\right)_{4} \mathrm{~F} \\
\text { residues after TGA. }\end{array}$ & S11 \\
\hline Table S5 & $\begin{array}{l}\text { Calculation of dipole moment for } \mathrm{TiO}_{6} \text { and } \mathrm{SeO}_{3} \text { polyhedra in } \\
\mathrm{Na}_{3} \mathrm{Ti}_{3} \mathrm{O}_{3}\left(\mathrm{SeO}_{3}\right)_{4} \mathrm{~F} \text { and } \mathrm{Ag}_{3} \mathrm{Ti}_{3} \mathrm{O}_{3}\left(\mathrm{SeO}_{3}\right)_{4} \mathrm{~F}\end{array}$ & S12 \\
\hline Table S6 & $\begin{array}{l}\text { State energies }(\mathrm{eV}) \text { of the highest valence band }(\mathrm{H}-\mathrm{VB}) \text { highest valence } \\
\text { band }(\mathrm{H}-\mathrm{VB}) \text { and the highest valence band }(\mathrm{H}-\mathrm{VB}) \text { of } \\
\mathrm{Na}_{3} \mathrm{Ti}_{3} \mathrm{O}_{3}\left(\mathrm{SeO}_{3}\right)_{4} \mathrm{~F} \text { and } \mathrm{Ag}_{3} \mathrm{Ti}_{3} \mathrm{O}_{3}\left(\mathrm{SeO}_{3}\right)_{4} \mathrm{~F} \text {. }\end{array}$ & S13 \\
\hline S1 & Computational Methods. & S14 \\
\hline
\end{tabular}




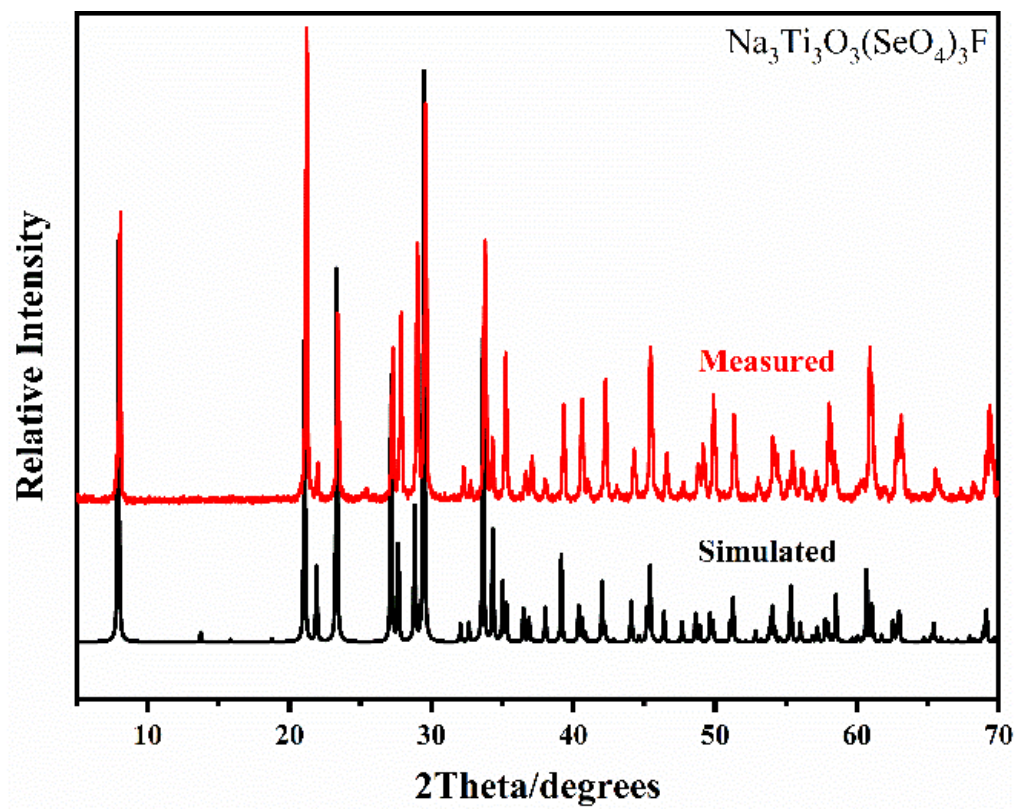

(a)

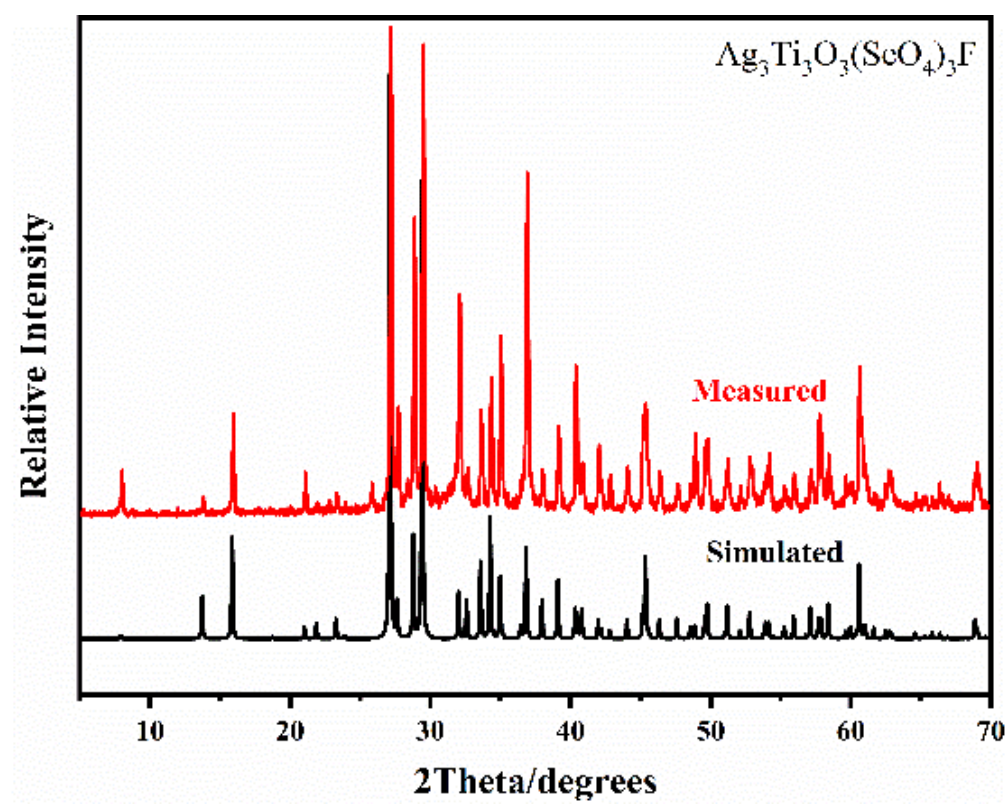

(b)

Figure S1. Simulated and experimental XRD powder patterns of $\mathrm{Na}_{3} \mathrm{Ti}_{3} \mathrm{O}_{3}\left(\mathrm{SeO}_{3}\right)_{4} \mathrm{~F}$ (a) and $\mathrm{Ag}_{3} \mathrm{Ti}_{3} \mathrm{O}_{3}\left(\mathrm{SeO}_{3}\right)_{4} \mathrm{~F}(\mathrm{~b})$. 


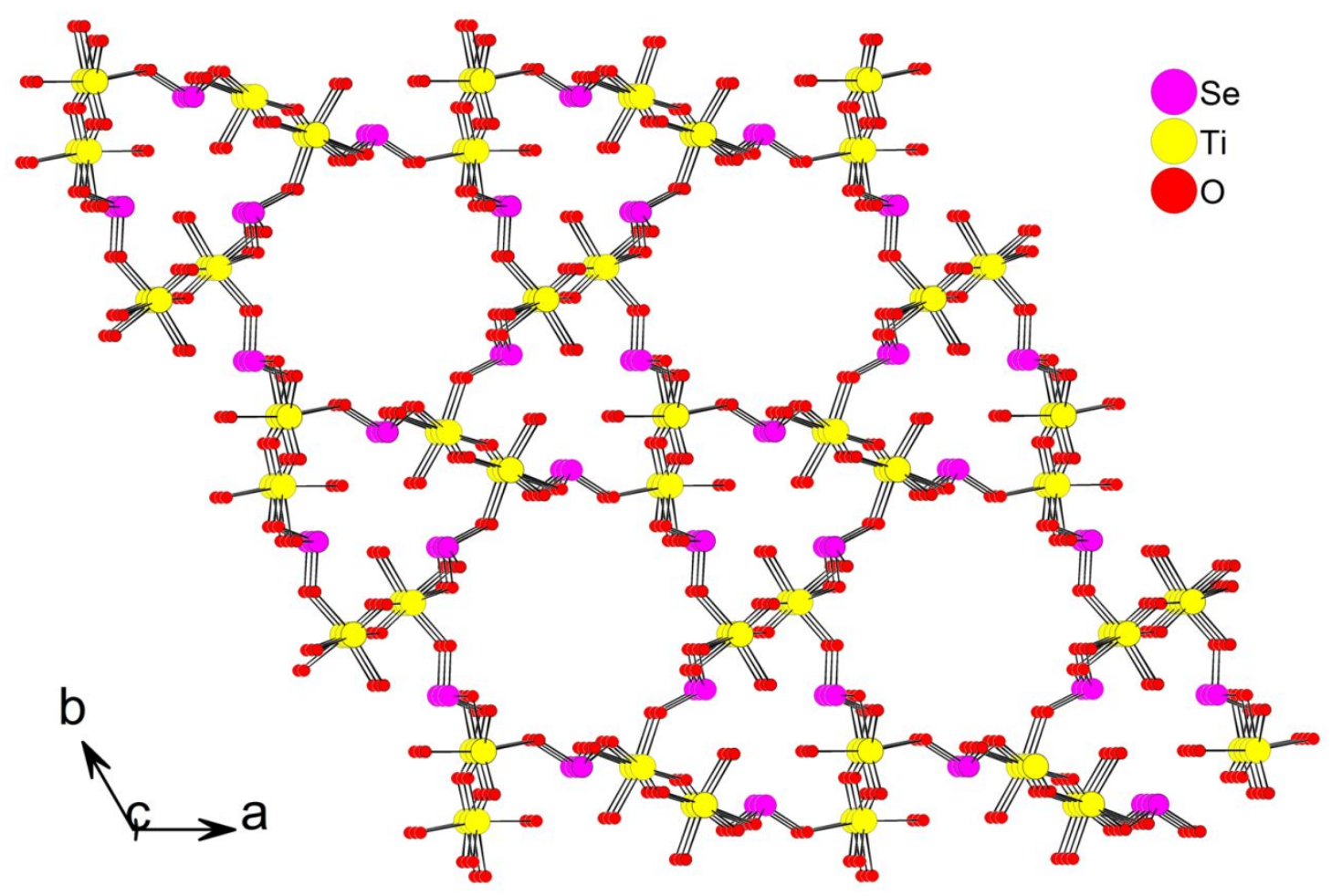

Figure S2. The 3D framework formed by $\mathrm{TiO}_{6}$ octahedral chains and $\mathrm{Se}(1) \mathrm{O}_{3}$ groups. 


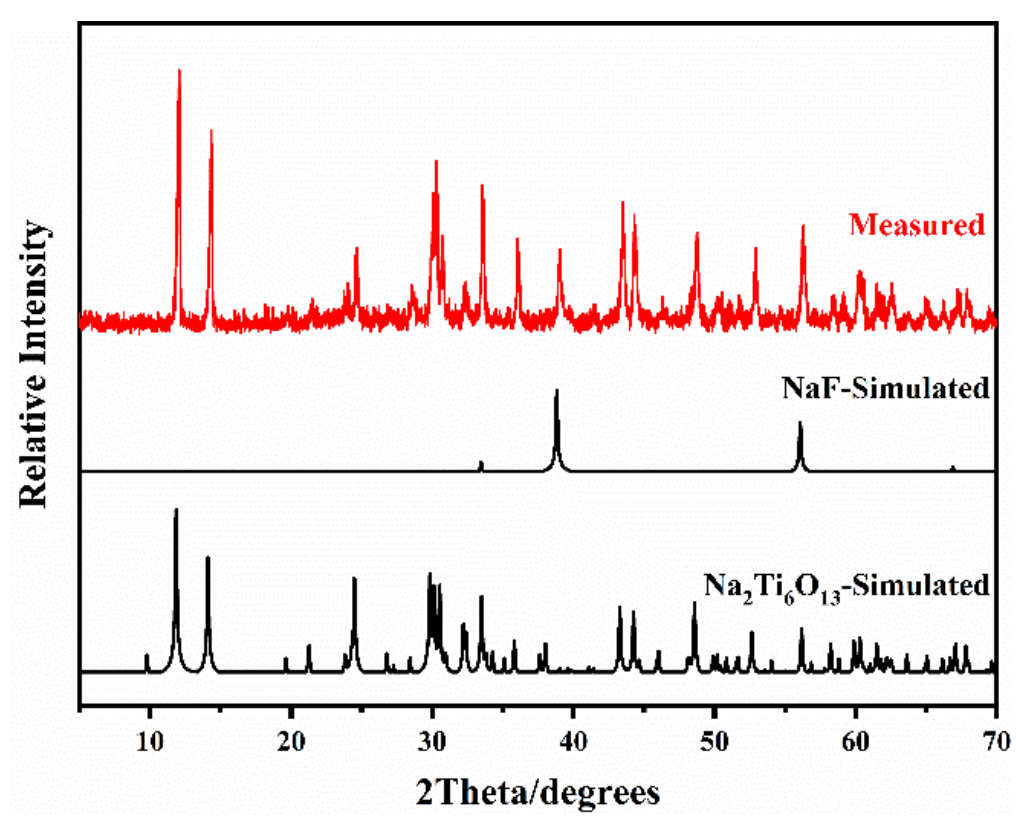

(a)

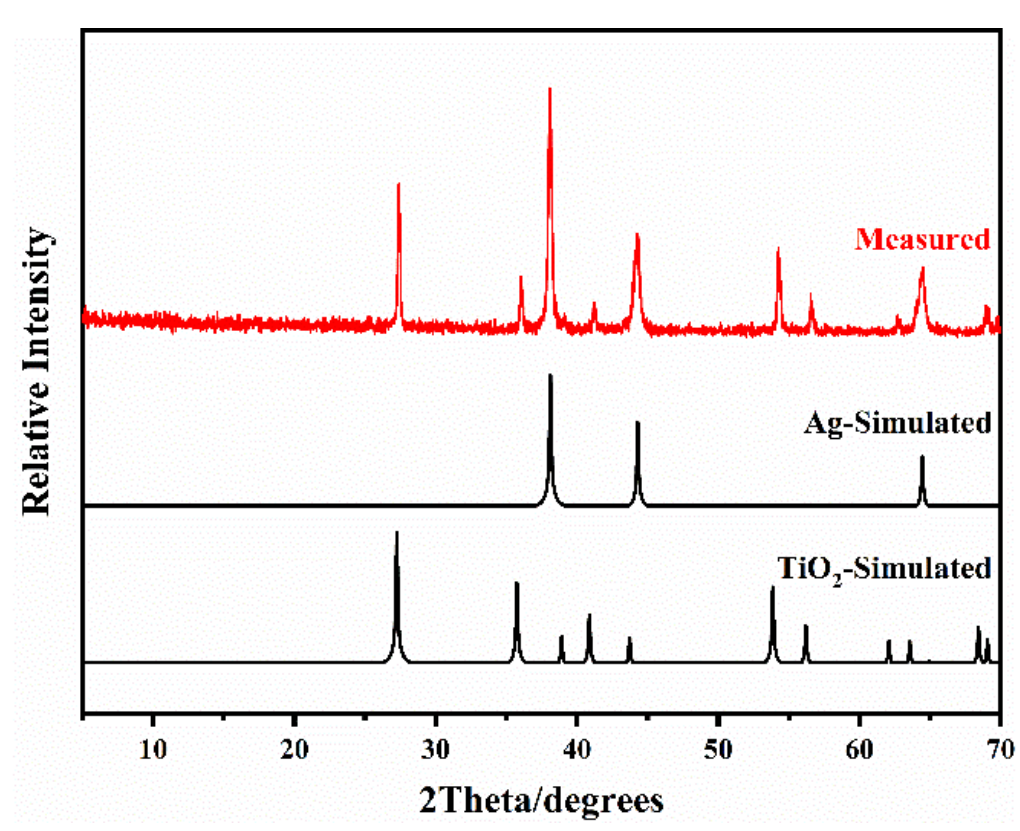

(b)

Figure S3. The measured XRD powder patterns for the residuals of $\mathrm{Na}_{3} \mathrm{Ti}_{3} \mathrm{O}_{3}\left(\mathrm{SeO}_{3}\right)_{4} \mathrm{~F}$ (a) and $\mathrm{Ag}_{3} \mathrm{Ti}_{3} \mathrm{O}_{3}\left(\mathrm{SeO}_{3}\right)_{4} \mathrm{~F}$ (b) after TGA, and the simulated patterns for $\mathrm{NaF}, \mathrm{Na}_{2} \mathrm{Ti}_{6} \mathrm{O}_{13}, \mathrm{Ag}$ and $\mathrm{TiO}_{2}$. 
Table S1. Atomic coordinates $\left(\times 10^{4}\right)$ and equivalent isotropic displacement parameters $\left(\AA^{2} \times 10^{3}\right)$ for $\mathrm{Na}_{3} \mathrm{Ti}_{3} \mathrm{O}_{3}\left(\mathrm{SeO}_{3}\right)_{4} \mathrm{~F}$ and $\mathrm{Ag}_{3} \mathrm{Ti}_{3} \mathrm{O}_{3}\left(\mathrm{SeO}_{3}\right)_{4} \mathrm{~F}$. $\mathrm{U}_{\text {eq }}$ is defined as $1 / 3$ of the trace of the orthogonalised $\mathrm{U}_{\mathrm{ij}}$ tensor.

\begin{tabular}{cccccc}
\hline & $\mathbf{A t o m}$ & $\mathbf{x}$ & $\mathbf{y}$ & $\mathbf{z}$ & $\mathbf{U}(\mathbf{e q})$ \\
\hline & $\mathrm{Se}(1)$ & $7876(2)$ & $5573(2)$ & $5431(9)$ & $8(1)$ \\
& $\mathrm{Na}(1)$ & $10630(13)$ & $8094(15)$ & $5820(40)$ & $34(4)$ \\
& $\mathrm{Se}(2)$ & 3333 & 6667 & $4989(11)$ & $8(1)$ \\
& $\mathrm{Ti}(1)$ & $5566(4)$ & $6043(4)$ & $5543(14)$ & $7(1)$ \\
$\mathbf{N a}_{\mathbf{3}} \mathbf{T i}_{\mathbf{3}} \mathbf{O}_{\mathbf{3}}\left(\mathbf{S e O}_{\mathbf{3}} \mathbf{3}_{\mathbf{4}} \mathbf{F}\right.$ & $\mathrm{O}(5)$ & $5182(19)$ & $4760(18)$ & $7400(50)$ & $9(4)$ \\
& $\mathrm{O}(4)$ & $3969(19)$ & $8014(18)$ & $6490(50)$ & $13(5)$ \\
& $\mathrm{O}(1)$ & $7187(17)$ & $6380(18)$ & $4720(50)$ & $12(5)$ \\
& $\mathrm{O}(2)$ & $9070(20)$ & $6321(19)$ & $3430(50)$ & $11(5)$ \\
& $\mathrm{O}(3)$ & $8460(20)$ & $6140(20)$ & $8330(50)$ & $16(5)$ \\
& $\mathrm{F}(1)$ & 10000 & 10000 & $8700(200)$ & $106(6)$ \\
\hline & $\mathrm{Se}(1)$ & $2165(1)$ & $4452(1)$ & $4564(3)$ & $4(1)$ \\
& $\mathrm{Ag}(1)$ & $-584(1)$ & $1927(1)$ & $4296(3)$ & $32(1)$ \\
& $\mathrm{Se}(2)$ & 6667 & 3333 & $5006(4)$ & $4(1)$ \\
& $\mathrm{Ti}(1)$ & $4456(2)$ & $3961(2)$ & $4462(5)$ & $6(1)$ \\
$\mathbf{A g}_{\mathbf{3}} \mathbf{T i}_{\mathbf{3}} \mathbf{O}_{\mathbf{3}} \mathbf{( S e O}_{\mathbf{3}} \mathbf{S}_{\mathbf{4}} \mathbf{F}$ & $\mathrm{O}(5)$ & $4813(9)$ & $5223(8)$ & $2575(19)$ & $11(2)$ \\
& $\mathrm{O}(4)$ & $6025(9)$ & $1993(8)$ & $3536(19)$ & $12(2)$ \\
& $\mathrm{O}(1)$ & $2826(8)$ & $3610(8)$ & $5265(18)$ & $11(2)$ \\
& $\mathrm{O}(2)$ & $963(9)$ & $3703(9)$ & $6568(17)$ & $10(2)$ \\
& $\mathrm{O}(3)$ & $1560(10)$ & $3893(10)$ & $1689(17)$ & $13(2)$ \\
& $\mathrm{F}(1)$ & 0 & 0 & $2160(60)$ & $51(5)$ \\
\hline
\end{tabular}


Table S2. The bond lengths $(\AA)$ and calculated bond valences for $\mathrm{Na}_{3} \mathrm{Ti}_{3} \mathrm{O}_{3}\left(\mathrm{SeO}_{3}\right)_{4} \mathrm{~F}$ and $\mathrm{Ag}_{3} \mathrm{Ti}_{3} \mathrm{O}_{3}\left(\mathrm{SeO}_{3}\right)_{4} \mathrm{~F}$.

\begin{tabular}{|c|c|c|c|c|}
\hline Compound & Bond & Bond-length & Bond-valence & BVS \\
\hline \multirow{20}{*}{$\mathrm{Na}_{3} \mathrm{Ti}_{3} \mathrm{O}_{3}\left(\mathrm{SeO}_{3}\right)_{4} \mathrm{~F}$} & $\mathrm{Se}(1)-\mathrm{O}(3)$ & $1.69(3)$ & 1.387 & \multirow{3}{*}{4.051} \\
\hline & $\mathrm{Se}(1)-\mathrm{O}(2)$ & $1.70(2)$ & 1.350 & \\
\hline & $\mathrm{Se}(1)-\mathrm{O}(1)$ & $1.71(2)$ & 1.314 & \\
\hline & $\mathrm{Na}(1)-\mathrm{O}(3) \# 2$ & $2.37(3)$ & 0.216 & \multirow{8}{*}{0.729} \\
\hline & $\mathrm{Na}(1)-\mathrm{O}(1) \# 3$ & $2.42(3)$ & 0.189 & \\
\hline & $\mathrm{Na}(1)-\mathrm{O}(2)$ & $2.49(3)$ & 0.156 & \\
\hline & $\mathrm{Na}(1)-\mathrm{O}(2) \# 3$ & $2.73(3)$ & 0.082 & \\
\hline & $\mathrm{Na}(1)-\mathrm{O}(3)$ & $2.97(3)$ & 0.043 & \\
\hline & $\mathrm{Na}(1)-\mathrm{F}(1) \# 4$ & $3.14(4)$ & 0.019 & \\
\hline & $\mathrm{Na}(1)-\mathrm{F}(1)$ & $3.30(5)$ & 0.012 & \\
\hline & $\mathrm{Na}(1)-\mathrm{O}(1) \# 2$ & $3.44(3)$ & 0.012 & \\
\hline & $\mathrm{Se}(2)-\mathrm{O}(4)$ & $1.69(2)$ & 1.387 & \multirow{3}{*}{4.161} \\
\hline & $\mathrm{Se}(2)-\mathrm{O}(4) \# 6$ & $1.69(2)$ & 1.387 & \\
\hline & $\mathrm{Se}(2)-\mathrm{O}(4) \# 7$ & $1.69(2)$ & 1.387 & \\
\hline & $\operatorname{Ti}(1)-\mathrm{O}(5)$ & $1.76(2)$ & 1.160 & \multirow{6}{*}{4.099} \\
\hline & $\mathrm{Ti}(1)-\mathrm{O}(5) \# 8$ & $1.93(2)$ & 0.733 & \\
\hline & $\operatorname{Ti}(1)-\mathrm{O}(1)$ & $1.95(2)$ & 0.694 & \\
\hline & $\mathrm{Ti}(1)-\mathrm{O}(4) \# 7$ & $2.01(2)$ & 0.590 & \\
\hline & $\mathrm{Ti}(1)-\mathrm{O}(2) \# 9$ & $2.03(2)$ & 0.559 & \\
\hline & $\mathrm{Ti}(1)-\mathrm{O}(3) \# 1$ & $2.19(3)$ & 0.363 & \\
\hline \multirow{18}{*}{$\mathrm{Ag}_{3} \mathrm{Ti}_{3} \mathrm{O}_{3}\left(\mathrm{SeO}_{3}\right)_{4} \mathrm{~F}$} & $\mathrm{Se}(1)-\mathrm{O}(3)$ & $1.681(10)$ & 1.421 & \multirow{3}{*}{3.990} \\
\hline & $\mathrm{Se}(1)-\mathrm{O}(2)$ & $1.714(9)$ & 1.300 & \\
\hline & $\mathrm{Se}(1)-\mathrm{O}(1)$ & $1.723(9)$ & 1.269 & \\
\hline & $\mathrm{Ag}(1)-\mathrm{O}(3) \# 1$ & $2.412(10)$ & 0.193 & \multirow{8}{*}{0.675} \\
\hline & $\mathrm{Ag}(1)-\mathrm{O}(2)$ & $2.464(10)$ & 0.168 & \\
\hline & $\mathrm{Ag}(1)-\mathrm{O}(1) \# 2$ & $2.481(9)$ & 0.160 & \\
\hline & $\mathrm{Ag}(1)-\mathrm{O}(2) \# 2$ & $2.804(10)$ & 0.067 & \\
\hline & $\operatorname{Ag}(1)-\mathrm{O}(3)$ & $2.987(11)$ & 0.041 & \\
\hline & $\operatorname{Ag}(1)-F(1)$ & $3.141(11)$ & 0.019 & \\
\hline & $\mathrm{Ag}(1)-\mathrm{F}(1) \# 3$ & $3.297(14)$ & 0.013 & \\
\hline & $\operatorname{Ag}(1)-\mathrm{O}(1) \# 1$ & $3.385(10)$ & 0.014 & \\
\hline & $\mathrm{Se}(2)-\mathrm{O}(4)$ & $1.684(10)$ & 1.410 & \multirow{3}{*}{4.230} \\
\hline & $\mathrm{Se}(2)-\mathrm{O}(4) \# 4$ & $1.684(10)$ & 1.410 & \\
\hline & $\mathrm{Se}(2)-\mathrm{O}(4) \# 5$ & $1.684(10)$ & 1.410 & \\
\hline & $\operatorname{Ti}(1)-\mathrm{O}(5)$ & $1.757(10)$ & 1.170 & \multirow{4}{*}{4.119} \\
\hline & $\mathrm{Ti}(1)-\mathrm{O}(5) \# 6$ & $1.912(11)$ & 0.769 & \\
\hline & $\operatorname{Ti}(1)-\mathrm{O}(1)$ & $1.963(9)$ & 0.670 & \\
\hline & $\mathrm{Ti}(1)-\mathrm{O}(4) \# 5$ & $2.000(10)$ & 0.607 & \\
\hline
\end{tabular}




\begin{tabular}{|l|c|c|c|c|}
\hline & $\operatorname{Ti}(1)-\mathrm{O}(2) \# 7$ & $2.047(9)$ & 0.534 & \multirow{2}{*}{} \\
\cline { 2 - 4 } & $\operatorname{Ti}(1)-\mathrm{O}(3) \# 8$ & $2.184(10)$ & 0.369 & \\
\hline
\end{tabular}

Symmetry transformations used to generate equivalent atoms:

For $\mathrm{Na}_{3} \mathrm{Ti}_{3} \mathrm{O}_{3}\left(\mathrm{SeO}_{3}\right)_{4} \mathrm{~F}$ :

$\begin{array}{llcr}\# 1 \mathrm{y},-\mathrm{x}+\mathrm{y}+1, \mathrm{z}-1 / 2 & \# 2 \mathrm{x}-\mathrm{y}+1, \mathrm{x}, \mathrm{z}-1 / 2 & \# 3 \mathrm{x}-\mathrm{y}+1, \mathrm{x}, \mathrm{z}+1 / 2 & \# 4-\mathrm{x}+2,-\mathrm{y}+2, \mathrm{z}-1 / 2 \\ \# 6-\mathrm{y}+1, \mathrm{x}-\mathrm{y}+1, \mathrm{z} & \# 7-\mathrm{x}+\mathrm{y},-\mathrm{x}+1, \mathrm{z} & \# 8-\mathrm{x}+1,-\mathrm{y}+1, \mathrm{z}-1 / 2 & \# 9 \mathrm{y},-\mathrm{x}+\mathrm{y}+1, \mathrm{z}+1 / 2\end{array}$

For $\mathrm{Ag}_{3} \mathrm{Ti}_{3} \mathrm{O}_{3}\left(\mathrm{SeO}_{3}\right)_{4} \mathrm{~F}$ :

\#1 x-y,x,z+1/2 \#2 x-y,x,z-1/2 \#3-x,-y,z+1/2 \#4-y+1,x-y,z \#5-x+y+1,$\mathrm{x}+1, \mathrm{z}$

$\# 6-x+1,-y+1, z+1 / 2 \quad \# 7 y,-x+y, z-1 / 2 \quad \# 8 y,-x+y, z+1 / 2$ 
Table S3. The bond angles (deg.) for $\mathrm{Na}_{3} \mathrm{Ti}_{3} \mathrm{O}_{3}\left(\mathrm{SeO}_{3}\right)_{4} \mathrm{~F}$ and $\mathrm{Ag}_{3} \mathrm{Ti}_{3} \mathrm{O}_{3}\left(\mathrm{SeO}_{3}\right)_{4} \mathrm{~F}$.

\begin{tabular}{|c|c|c|c|}
\hline \multicolumn{2}{|c|}{$\mathrm{Na}_{3} \mathrm{Ti}_{3} \mathrm{O}_{3}\left(\mathrm{SeO}_{3}\right)_{4} \mathrm{~F}$} & \multicolumn{2}{|c|}{$\mathrm{Ag}_{3} \mathrm{Ti}_{3} \mathrm{O}_{3}\left(\mathrm{SeO}_{3}\right)_{4} \mathrm{~F}$} \\
\hline $\mathrm{O}(3)-\mathrm{Se}(1)-\mathrm{O}(2)$ & $103.2(13)$ & $\mathrm{O}(3)-\mathrm{Se}(1)-\mathrm{O}(2)$ & $102.1(5)$ \\
\hline $\mathrm{O}(3)-\mathrm{Se}(1)-\mathrm{O}(1)$ & $100.6(12)$ & $\mathrm{O}(3)-\mathrm{Se}(1)-\mathrm{O}(1)$ & $101.0(5)$ \\
\hline $\mathrm{O}(2)-\mathrm{Se}(1)-\mathrm{O}(1)$ & $96.8(10)$ & $\mathrm{O}(2)-\mathrm{Se}(1)-\mathrm{O}(1)$ & $96.2(4)$ \\
\hline $\mathrm{O}(3) \# 2-\mathrm{Na}(1)-\mathrm{O}(1) \# 3$ & $105.3(10)$ & $\mathrm{O}(3) \# 1-\mathrm{Ag}(1)-\mathrm{O}(2)$ & $100.1(3)$ \\
\hline $\mathrm{O}(3) \# 2-\mathrm{Na}(1)-\mathrm{O}(2)$ & $98.2(11)$ & $\mathrm{O}(3) \# 1-\mathrm{Ag}(1)-\mathrm{O}(1) \# 2$ & $103.1(3)$ \\
\hline $\mathrm{O}(1) \# 3-\mathrm{Na}(1)-\mathrm{O}(2)$ & $102.1(10)$ & $\mathrm{O}(2)-\mathrm{Ag}(1)-\mathrm{O}(1) \# 2$ & $101.6(3)$ \\
\hline $\mathrm{O}(3) \# 2-\mathrm{Na}(1)-\mathrm{O}(2) \# 3$ & $65.3(9)$ & $\mathrm{O}(3) \# 1-\mathrm{Ag}(1)-\mathrm{O}(2) \# 2$ & $63.5(3)$ \\
\hline $\mathrm{O}(1) \# 3-\mathrm{Na}(1)-\mathrm{O}(2) \# 3$ & $59.0(8)$ & $\mathrm{O}(2)-\mathrm{Ag}(1)-\mathrm{O}(2) \# 2$ & $145.1(3)$ \\
\hline $\mathrm{O}(2)-\mathrm{Na}(1)-\mathrm{O}(2) \# 3$ & $146.5(9)$ & $\mathrm{O}(1) \# 2-\mathrm{Ag}(1)-\mathrm{O}(2) \# 2$ & $57.5(3)$ \\
\hline $\mathrm{O}(3) \# 2-\mathrm{Na}(1)-\mathrm{O}(3)$ & $142.4(10)$ & $\mathrm{O}(3) \# 1-\mathrm{Ag}(1)-\mathrm{O}(3) 1$ & $41.4(3)$ \\
\hline $\mathrm{O}(1) \# 3-\mathrm{Na}(1)-\mathrm{O}(3)$ & $59.6(8)$ & $\mathrm{O}(2)-\mathrm{Ag}(1)-\mathrm{O}(3)$ & $57.0(3)$ \\
\hline $\mathrm{O}(2)-\mathrm{Na}(1)-\mathrm{O}(3)$ & $57.5(8)$ & $\mathrm{O}(1) \# 2-\mathrm{Ag}(1)-\mathrm{O}(3)$ & $58.9(3)$ \\
\hline $\mathrm{O}(2) \# 3-\mathrm{Na}(1)-\mathrm{O}(3)$ & $117.9(10)$ & $\mathrm{O}(2) \# 2-\mathrm{Ag}(1)-\mathrm{O}(3)$ & $115.9(3)$ \\
\hline $\mathrm{O}(3) \# 2-\mathrm{Na}(1)-\mathrm{F}(1) \# 4$ & $102.5(15)$ & $\mathrm{O}(3) \# 1-\mathrm{Ag}(1)-\mathrm{F}(1)$ & $126.9(3)$ \\
\hline $\mathrm{O}(1) \# 3-\mathrm{Na}(1)-\mathrm{F}(1) \# 4$ & $143(2)$ & $\mathrm{O}(2)-\mathrm{Ag}(1)-\mathrm{F}(1)$ & $120.3(3)$ \\
\hline $\mathrm{O}(2)-\mathrm{Na}(1)-\mathrm{F}(1) \# 4$ & $97.5(14)$ & $\mathrm{O}(1) \# 2-\mathrm{Ag}(1)-\mathrm{F}(1)$ & $101.0(6)$ \\
\hline $\mathrm{O}(2) \# 3-\mathrm{Na}(1)-\mathrm{F}(1) \# 4$ & $114.0(11)$ & $\mathrm{O}(2) \# 2-\mathrm{Ag}(1)-\mathrm{F}(1)$ & $92.6(4)$ \\
\hline $\mathrm{O}(3)-\mathrm{Na}(1)-\mathrm{F}(1) \# 4$ & $108.4(10)$ & $\mathrm{O}(3)-\mathrm{Ag}(1)-\mathrm{F}(1)$ & $91.3(3)$ \\
\hline $\mathrm{O}(3) \# 2-\mathrm{Na}(1)-\mathrm{F}(1)$ & $130.1(11)$ & $\mathrm{O}(3) \# 1-\mathrm{Ag}(1)-\mathrm{F}(1) \# 3$ & $99.2(4)$ \\
\hline $\mathrm{O}(1) \# 3-\mathrm{Na}(1)-\mathrm{F}(1)$ & $95.3(19)$ & $\mathrm{O}(2)-\mathrm{Ag}(1)-\mathrm{F}(1) \# 3$ & $95.6(4)$ \\
\hline $\mathrm{O}(2)-\mathrm{Na}(1)-\mathrm{F}(1)$ & $121.5(9)$ & $\mathrm{O}(1) \# 2-\mathrm{Ag}(1)-\mathrm{F}(1) \# 3$ & $148.8(5)$ \\
\hline $\mathrm{O}(2) \# 3-\mathrm{Na}(1)-\mathrm{F}(1)$ & $89.4(12)$ & $\mathrm{O}(2) \# 2-\mathrm{Ag}(1)-\mathrm{F}(1) \# 3$ & $116.6(3)$ \\
\hline $\mathrm{O}(3)-\mathrm{Na}(1)-\mathrm{F}(1)$ & $87.2(10)$ & $\mathrm{O}(3)-\mathrm{Ag}(1)-\mathrm{F}(1) \# 3$ & $112.6(2)$ \\
\hline $\mathrm{F}(1) \# 4-\mathrm{Na}(1)-\mathrm{F}(1)$ & $47.9(3)$ & $\mathrm{F}(1)-\operatorname{Ag}(1)-\mathrm{F}(1) \# 3$ & $47.85(5)$ \\
\hline $\mathrm{O}(3) \# 2-\mathrm{Na}(1)-\mathrm{O}(1) \# 2$ & $49.2(8)$ & $\mathrm{O}(3) \# 1-\mathrm{Ag}(1)-\mathrm{O}(1) \# 1$ & $50.5(3)$ \\
\hline $\mathrm{O}(1) \# 3-\mathrm{Na}(1)-\mathrm{O}(1) \# 2$ & $125.6(10)$ & $\mathrm{O}(2)-\mathrm{Ag}(1)-\mathrm{O}(1) \# 1$ & $53.3(3)$ \\
\hline $\mathrm{O}(2)-\mathrm{Na}(1)-\mathrm{O}(1) \# 2$ & $52.0(8)$ & $\mathrm{O}(1) \# 2-\mathrm{Ag}(1)-\mathrm{O}(1) \# 1$ & $125.4(3)$ \\
\hline $\mathrm{O}(2) \# 3-\mathrm{Na}(1)-\mathrm{O}(1) \# 2$ & $113.6(8)$ & $\mathrm{O}(2) \# 2-\mathrm{Ag}(1)-\mathrm{O}(1) \# 1$ & $113.1(2)$ \\
\hline $\mathrm{O}(3)-\mathrm{Na}(1)-\mathrm{O}(1) \# 2$ & $108.5(8)$ & $\mathrm{O}(3)-\mathrm{Ag}(1)-\mathrm{O}(1) \# 1$ & $109.1(2)$ \\
\hline $\mathrm{F}(1) \# 4-\mathrm{Na}(1)-\mathrm{O}(1) \# 2$ & $91.1(19)$ & $\mathrm{F}(1)-\mathrm{Ag}(1)-\mathrm{O}(1) \# 1$ & $133.5(5)$ \\
\hline $\mathrm{F}(1)-\mathrm{Na}(1)-\mathrm{O}(1) \# 2$ & $138.9(18)$ & $\mathrm{F}(1) \# 3-\mathrm{Ag}(1)-\mathrm{O}(1) \# 1$ & $85.7(5)$ \\
\hline $\mathrm{O}(4)-\mathrm{Se}(2)-\mathrm{O}(4) \# 6$ & $100.1(11)$ & $\mathrm{O}(4)-\mathrm{Se}(2)-\mathrm{O}(4) \# 4$ & $100.8(4)$ \\
\hline $\mathrm{O}(4)-\mathrm{Se}(2)-\mathrm{O}(4) \# 7$ & $100.1(11)$ & $\mathrm{O}(4)-\mathrm{Se}(2)-\mathrm{O}(4) \# 5$ & $100.8(4)$ \\
\hline $\mathrm{O}(4) \# 6-\mathrm{Se}(2)-\mathrm{O}(4) \# 7$ & $100.1(11)$ & $\mathrm{O}(4) \# 4-\mathrm{Se}(2)-\mathrm{O}(4) \# 5$ & $100.8(4)$ \\
\hline $\mathrm{O}(5)-\mathrm{Ti}(1)-\mathrm{O}(5) \# 8$ & $98.0(5)$ & $\mathrm{O}(5)-\mathrm{Ti}(1)-\mathrm{O}(5) \# 6$ & $98.0(2)$ \\
\hline $\mathrm{O}(5)-\mathrm{Ti}(1)-\mathrm{O}(1)$ & $95.6(10)$ & $\mathrm{O}(5)-\mathrm{Ti}(1)-\mathrm{O}(1)$ & $95.1(4)$ \\
\hline $\mathrm{O}(5) \# 8-\mathrm{Ti}(1)-\mathrm{O}(1)$ & $97.0(10)$ & $\mathrm{O}(5) \# 6-\mathrm{Ti}(1)-\mathrm{O}(1)$ & $97.0(4)$ \\
\hline $\mathrm{O}(5)-\mathrm{Ti}(1)-\mathrm{O}(4) \# 7$ & $92.3(10)$ & $\mathrm{O}(5)-\mathrm{Ti}(1)-\mathrm{O}(4) \# 5$ & $93.0(5)$ \\
\hline $\mathrm{O}(5) \# 8-\mathrm{Ti}(1)-\mathrm{O}(4) \# 7$ & $89.2(10)$ & $\mathrm{O}(5) \# 6-\mathrm{Ti}(1)-\mathrm{O}(4) \# 5$ & $89.5(4)$ \\
\hline $\mathrm{O}(1)-\mathrm{Ti}(1)-\mathrm{O}(4) \# 7$ & $169.2(9)$ & $\mathrm{O}(1)-\mathrm{Ti}(1)-\mathrm{O}(4) \# 5$ & $168.8(4)$ \\
\hline $\mathrm{O}(5)-\mathrm{Ti}(1)-\mathrm{O}(2) \# 9$ & $96.2(11)$ & $\mathrm{O}(5)-\mathrm{Ti}(1)-\mathrm{O}(2) \# 7$ & $95.8(4)$ \\
\hline
\end{tabular}




\begin{tabular}{llll}
$\mathrm{O}(5) \# 8-\mathrm{Ti}(1)-\mathrm{O}(2) \# 9$ & $164.9(10)$ & $\mathrm{O}(5) \# 6-\mathrm{Ti}(1)-\mathrm{O}(2) \# 7$ & $165.4(4)$ \\
$\mathrm{O}(1)-\mathrm{Ti}(1)-\mathrm{O}(2) \# 9$ & $86.9(10)$ & $\mathrm{O}(1)-\mathrm{Ti}(1)-\mathrm{O}(2) \# 7$ & $86.5(4)$ \\
$\mathrm{O}(4) \# 7-\mathrm{Ti}(1)-\mathrm{O}(2) \# 9$ & $85.0(10)$ & $\mathrm{O}(4) \# 5-\mathrm{Ti}(1)-\mathrm{O}(2) \# 7$ & $85.0(4)$ \\
$\mathrm{O}(5)-\mathrm{Ti}(1)-\mathrm{O}(3) \# 1$ & $176.8(10)$ & $\mathrm{O}(5)-\mathrm{Ti}(1)-\mathrm{O}(3) \# 8$ & $176.1(4)$ \\
$\mathrm{O}(5) \# 8-\mathrm{Ti}(1)-\mathrm{O}(3) \# 1$ & $84.3(10)$ & $\mathrm{O}(5) \# 6-\mathrm{Ti}(1)-\mathrm{O}(3) \# 8$ & $84.9(4)$ \\
$\mathrm{O}(1)-\mathrm{Ti}(1)-\mathrm{O}(3) \# 1$ & $81.9(10)$ & $\mathrm{O}(1)-\mathrm{Ti}(1)-\mathrm{O}(3) \# 8$ & $82.0(4)$ \\
$\mathrm{O}(4) \# 7-\mathrm{Ti}(1)-\mathrm{O}(3) \# 1$ & $89.9(9)$ & $\mathrm{O}(4) \# 5-\mathrm{Ti}(1)-\mathrm{O}(3) \# 8$ & $89.5(4)$ \\
$\mathrm{O}(2) \# 9-\mathrm{Ti}(1)-\mathrm{O}(3) \# 1$ & $81.8(10)$ & $\mathrm{O}(2) \# 7-\mathrm{Ti}(1)-\mathrm{O}(3) \# 8$ & $81.5(4)$ \\
\hline
\end{tabular}

Symmetry transformations used to generate equivalent atoms:

For $\mathrm{Na}_{3} \mathrm{Ti}_{3} \mathrm{O}_{3}\left(\mathrm{SeO}_{3}\right)_{4} \mathrm{~F}$ :

$\begin{array}{llcc}\# 1 \mathrm{y},-\mathrm{x}+\mathrm{y}+1, \mathrm{z}-1 / 2 & \# 2 \mathrm{x}-\mathrm{y}+1, \mathrm{x}, \mathrm{z}-1 / 2 & \# 3 \mathrm{x}-\mathrm{y}+1, \mathrm{x}, \mathrm{z}+1 / 2 & \# 4-\mathrm{x}+2,-\mathrm{y}+2, \mathrm{z}-1 / 2 \\ \# 6-\mathrm{y}+1, \mathrm{x}-\mathrm{y}+1, \mathrm{z} & \# 7-\mathrm{x}+\mathrm{y},-\mathrm{x}+1, \mathrm{z} & \# 8-\mathrm{x}+1,-\mathrm{y}+1, \mathrm{z}-1 / 2 & \# 9 \mathrm{y},-\mathrm{x}+\mathrm{y}+1, \mathrm{z}+1 / 2\end{array}$

For $\mathrm{Ag}_{3} \mathrm{Ti}_{3} \mathrm{O}_{3}\left(\mathrm{SeO}_{3}\right)_{4} \mathrm{~F}$ :

\#1 x-y,x,z+1/2 \#2 x-y,x,z-1/2 \#3-x,-y,z+1/2 \#4-y+1,x-y,z \#5-x+y+1,$\mathrm{x}+1, \mathrm{z}$

$\# 6-x+1,-y+1, z+1 / 2 \quad \# 7 y,-x+y, z-1 / 2 \quad \# 8 y,-x+y, z+1 / 2$ 
Table S4. The EDS analyses for the residues of $\mathrm{Na}_{3} \mathrm{Ti}_{3} \mathrm{O}_{3}\left(\mathrm{SeO}_{3}\right)_{4} \mathrm{~F}$ and $\mathrm{Ag}_{3} \mathrm{Ti}_{3} \mathrm{O}_{3}\left(\mathrm{SeO}_{3}\right)_{4} \mathrm{~F}$ after TGA.

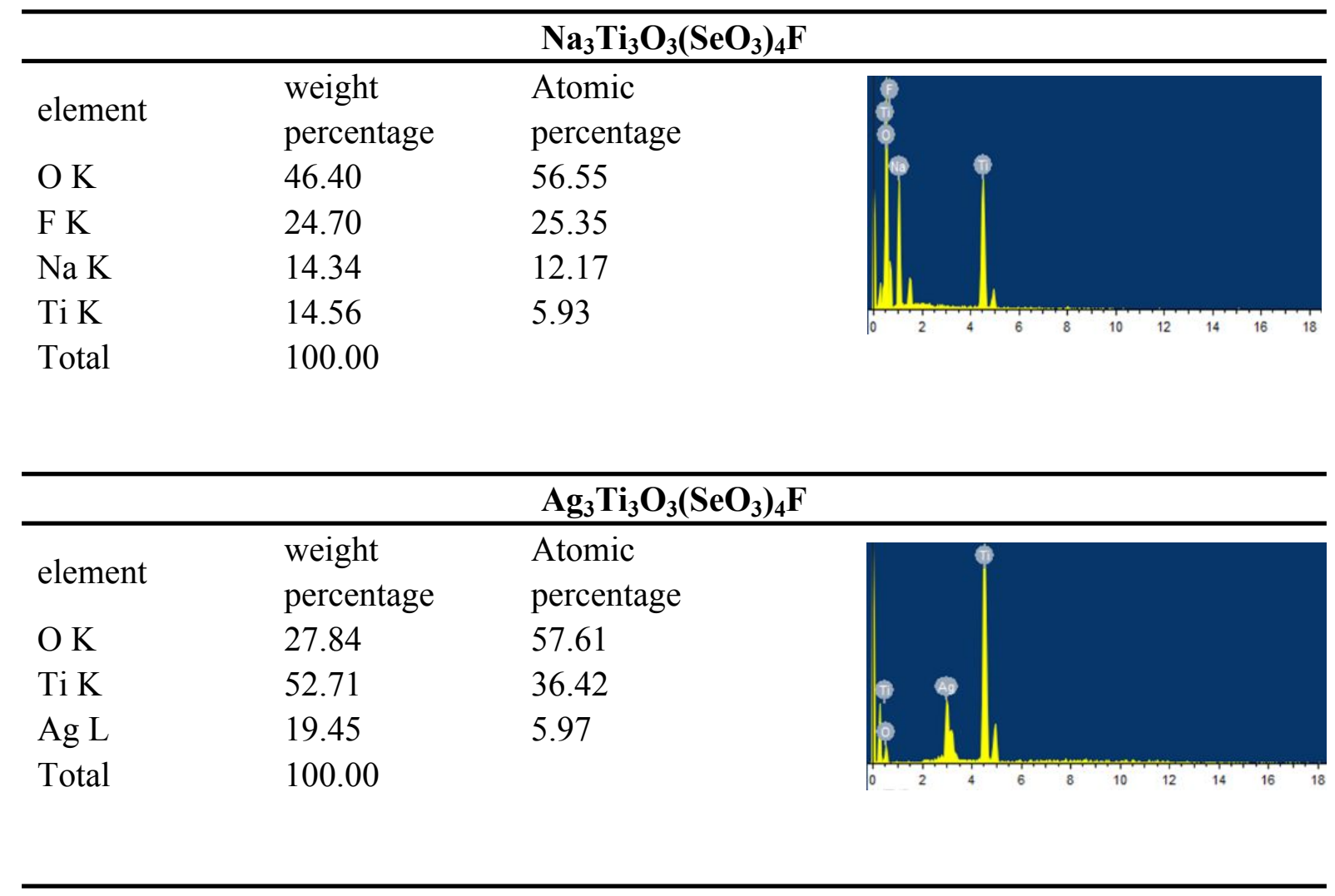


Table S5. Calculation of dipole moment for $\mathrm{TiO}_{6}$ and $\mathrm{SeO}_{3}$ polyhedra in $\mathrm{Na}_{3} \mathrm{Ti}_{3} \mathrm{O}_{3}\left(\mathrm{SeO}_{3}\right)_{4} \mathrm{~F}$ and $\mathrm{Ag}_{3} \mathrm{Ti}_{3} \mathrm{O}_{3}\left(\mathrm{SeO}_{3}\right)_{4} \mathrm{~F}(\mathrm{D}=$ Debyes).

\begin{tabular}{|c|c|c|c|c|}
\hline \multicolumn{5}{|c|}{$\mathrm{Na}_{3} \mathrm{Ti}_{3} \mathrm{O}_{3}\left(\mathrm{SeO}_{3}\right)_{4} \mathrm{~F}$} \\
\hline \multirow{2}{*}{ Polar unit } & \multicolumn{4}{|c|}{ Dipole moment (D) } \\
\hline & $\mathrm{x}$-component & $\mathrm{y}$-component & z-component & total magnitude \\
\hline $\mathrm{Ti}(1) \mathrm{O}_{6}$ & -3 & -2.87 & -0.2 & 4.16 \\
\hline $\operatorname{Ti}(1) \mathrm{O}_{6}$ & 3.99 & -1.17 & -0.2 & 4.16 \\
\hline $\mathrm{Ti}(1) \mathrm{O}_{6}$ & -0.98 & 4.03 & -0.2 & 4.15 \\
\hline $\operatorname{Ti}(1) \mathrm{O}_{6}$ & 0.98 & -4.04 & -0.2 & 4.16 \\
\hline $\mathrm{Ti}(1) \mathrm{O}_{6}$ & -3.99 & 1.17 & -0.2 & 4.16 \\
\hline $\operatorname{Ti}(1) \mathrm{O}_{6}$ & 3.01 & 2.87 & -0.2 & 4.16 \\
\hline $\mathrm{Se}(1) \mathrm{O}_{3}$ & -8.15 & 4.94 & 0.56 & 9.55 \\
\hline $\mathrm{Se}(1) \mathrm{O}_{3}$ & 8.36 & 4.58 & 0.56 & 9.55 \\
\hline $\mathrm{Se}(1) \mathrm{O}_{3}$ & -0.21 & -9.53 & 0.56 & 9.55 \\
\hline $\mathrm{Se}(1) \mathrm{O}_{3}$ & 0.21 & 9.54 & 0.56 & 9.55 \\
\hline $\mathrm{Se}(1) \mathrm{O}_{3}$ & -8.35 & -4.58 & 0.56 & 9.54 \\
\hline $\mathrm{Se}(1) \mathrm{O}_{3}$ & 8.15 & -4.95 & 0.56 & 9.55 \\
\hline $\mathrm{Se}(2) \mathrm{O}_{3}$ & 0 & 0 & 9.76 & 9.76 \\
\hline $\mathrm{Se}(2) \mathrm{O}_{3}$ & 0 & 0 & 9.76 & 9.76 \\
\hline $\begin{array}{c}\text { Net dipole } \\
\text { moment } \\
\text { (a unit cell) }\end{array}$ & 0.01 & 0 & 21.67 & 21.67 \\
\hline \multicolumn{5}{|c|}{$\mathrm{Ag}_{3} \mathrm{Ti}_{3} \mathrm{O}_{3}\left(\mathrm{SeO}_{3}\right)_{4} \mathrm{~F}$} \\
\hline \multirow{2}{*}{ Polar unit } & \multicolumn{4}{|c|}{ Dipole moment (D) } \\
\hline & X-component & y-component & z-component & total magnitude \\
\hline $\operatorname{Ti}(1) \mathrm{O}_{6}$ & -3.84 & -2.67 & -0.3 & 4.69 \\
\hline $\mathrm{Ti}(1) \mathrm{O}_{6}$ & 4.24 & -1.99 & -0.3 & 4.69 \\
\hline $\operatorname{Ti}(1) \mathrm{O}_{6}$ & -0.39 & 4.67 & -0.3 & 4.69 \\
\hline $\operatorname{Ti}(1) \mathrm{O}_{6}$ & 0.39 & -4.67 & -0.3 & 4.69 \\
\hline $\mathrm{Ti}(1) \mathrm{O}_{6}$ & -4.24 & 1.99 & -0.3 & 4.69 \\
\hline $\operatorname{Ti}(1) \mathrm{O}_{6}$ & 3.84 & 2.67 & -0.3 & 4.69 \\
\hline $\mathrm{Se}(1) \mathrm{O}_{3}$ & -8.08 & 5.36 & 0.71 & 9.72 \\
\hline $\mathrm{Se}(1) \mathrm{O}_{3}$ & -0.6 & -9.67 & 0.71 & 9.72 \\
\hline $\mathrm{Se}(1) \mathrm{O}_{3}$ & 8.68 & 4.32 & 0.71 & 9.72 \\
\hline $\mathrm{Se}(1) \mathrm{O}_{3}$ & 0.6 & 9.67 & 0.71 & 9.72 \\
\hline $\mathrm{Se}(1) \mathrm{O}_{3}$ & -8.68 & -4.32 & 0.71 & 9.72 \\
\hline $\mathrm{Se}(1) \mathrm{O}_{3}$ & 8.08 & -5.36 & 0.71 & 9.72 \\
\hline $\mathrm{Se}(2) \mathrm{O}_{3}$ & 0 & 0 & 9.94 & 9.94 \\
\hline $\mathrm{Se}(2) \mathrm{O}_{3}$ & 0 & 0 & 9.94 & 9.94 \\
\hline $\begin{array}{l}\text { Net dipole moment } \\
\text { (a unit cell) }\end{array}$ & 0 & 0 & 22.32 & 22.32 \\
\hline
\end{tabular}


Table S6. State energies (eV) of the highest valence band (H-VB) highest valence band (HVB) and the highest valence band $(\mathrm{H}-\mathrm{VB})$ of $\mathrm{Na}_{3} \mathrm{Ti}_{3} \mathrm{O}_{3}\left(\mathrm{SeO}_{3}\right)_{4} \mathrm{~F}$ and $\mathrm{Ag}_{3} \mathrm{Ti}_{3} \mathrm{O}_{3}\left(\mathrm{SeO}_{3}\right)_{4} \mathrm{~F}$.

\begin{tabular}{|l|l|l|l|}
\hline Compound & K-point & H-VB & L-CB \\
\hline \multirow{5}{*}{$\mathbf{N a}_{\mathbf{3}} \mathbf{T i}_{\mathbf{3}} \mathbf{O}_{\mathbf{3}}\left(\mathbf{S e O}_{\mathbf{3}}\right)_{\mathbf{4}} \mathbf{F}$} & $\mathrm{G}(0.000,0.000,0.000)$ & 0 & 2.77006 \\
\cline { 2 - 4 } & $\mathrm{A}(0.000,0.000,0.500)$ & -0.37604 & 2.94682 \\
\cline { 2 - 4 } & $\mathrm{H}(-0.333,0.667,0.500)$ & -0.36911 & 2.94373 \\
\cline { 2 - 4 } & $\mathrm{K}(-0.333,0.667,0.000)$ & -0.05303 & 2.77215 \\
\cline { 2 - 4 } & $\mathrm{G}(0.000,0.000,0.000)$ & 0 & 2.77006 \\
\cline { 2 - 4 } & $\mathrm{M}(0.000,0.500,0.000)$ & -0.04509 & 2.77186 \\
\cline { 2 - 4 } & $\mathrm{L}(0.000,0.500,0.500)$ & -0.36676 & 2.94096 \\
\cline { 2 - 4 } & $\mathrm{H}(-0.333,0.667,0.500)$ & -0.36911 & 2.94373 \\
\hline \multirow{5}{*}{$\mathbf{A g}_{\mathbf{3}} \mathbf{T i}_{\mathbf{3}} \mathbf{O}_{\mathbf{3}}\left(\mathbf{S e O}_{\mathbf{3}}\right)_{\mathbf{4}} \mathbf{F}$} & $\mathrm{G}(0.000,0.000,0.000)$ & 0 & 1.72015 \\
\cline { 2 - 4 } & $\mathrm{A}(0.000,0.000,0.500)$ & -0.20366 & 2.57972 \\
\cline { 2 - 4 } & $\mathrm{H}(-0.333,0.667,0.500)$ & -0.2078 & 2.53097 \\
\cline { 2 - 4 } & $\mathrm{K}(-0.333,0.667,0.000)$ & -0.03846 & 1.77663 \\
\cline { 2 - 4 } & $\mathrm{G}(0.000,0.000,0.000)$ & 0 & 2.72015 \\
\cline { 2 - 4 } & $\mathrm{M}(0.000,0.500,0.000)$ & -0.03602 & 2.53097 \\
\cline { 2 - 4 } & $\mathrm{L}(0.000,0.500,0.500)$ & -0.20616 & -0.2078 \\
\cline { 2 - 4 } & $\mathrm{H}(-0.333,0.667,0.500)$ & & \\
\hline
\end{tabular}




\section{Computational Methods.}

Single-crystal structural data of $\mathrm{Na}_{3} \mathrm{Ti}_{3} \mathrm{O}_{3}\left(\mathrm{SeO}_{3}\right)_{4} \mathrm{~F}$ and $\mathrm{Ag}_{3} \mathrm{Ti}_{3} \mathrm{O}_{3}\left(\mathrm{SeO}_{3}\right)_{4} \mathrm{~F}$ were directly used for the theoretical calculations. The electronic structures and optical properties were calculated by using a plane-wave pseudopotentials method within density functional theory (DFT) implemented in the total energy code of CASTEP. ${ }^{1-2}$ For the exchange-correlation functional, we chose Perdew-Burke-Ernzerhof (PBE) in the generalized gradient approximation (GGA). ${ }^{3}$ The interactions between the ionic cores and the electrons were described by the norm-conserving pseudopotential. ${ }^{4}$ The following valence-electron configurations were considered in the computation: O- $2 s^{2} 2 p^{4}, F-2 s^{2} 2 p^{5}$, Se- $4 s^{2} 4 p^{4}$, Ti$3 s^{2} 3 p^{6} 3 d^{2} 4 s^{2}, N a-2 s^{2} 2 p^{6} 3 s^{1}$, and Ag- $4 s^{2} 4 p^{6} 4 d^{10} 5 s^{1}$. The number of plane waves included in the basis sets was determined by a cutoff energy of $850 \mathrm{eV}$. Monkhorst-Pack k-point sampling of $2 \times 2 \times 6$ was used to perform numerical integration of the Brillouin zone for the two compounds. During the optical property calculations, about 400 empty bands were set to ensure the convergence of SHG coefficients.

The calculations of second-order NLO properties were based on length-gauge formalism within the independent particle approximation. ${ }^{5}$ The second-order NLO susceptibility can be expressed as

$\chi a b c \mathrm{~L}(-2 \omega ; \omega, \omega)=\chi a b c \mathrm{~L}, \mathrm{e}(-2 \omega ; \omega, \omega)+\chi a b c \mathrm{~L}, \mathrm{i}(-2 \omega ; \omega, \omega)$,

where the subscript $\mathrm{L}$ denotes the length gauge, $\chi a b c \mathrm{~L}$, e and $\chi a b c \mathrm{~L}$, i give the contributions to $\chi a b c$ Lfrom interband processes and intraband processes, respectively.

To show the distribution of the quantum states relevant to SHG process, the SHG-weighted electron density analysis was implemented. In the scheme, the considered SHG coefficient is "resolved" into each band/orbital, and based on the "band-resolved" method, each band/orbital's contribution to a specific SHG coefficient can be identified. Using the normalized effective SHG for each band/orbital as a weighting coefficient, and summing all the SHG-weighted bands/orbitals in VB or CB over the different $\mathrm{k}$ points in the space, the SHG-weighted electron density can be obtained. ${ }^{6}$ 


\section{References}

[1] Milman, V.; Winkler, B.; White, J. A.; Pickard, C. J.; Payne, M. C.; Akhmatskaya, E. V.; Nobes, R. H. Electronic Structure, Properties, and Phase Stability of Inorganic Crystals: A Pseudopotential Plane-Wave Study. Int. J. Quantum Chem. 2000, 77, 895910.

[2] Segall, M. D.; Lindan, P. J. D.; Probert, M. J.; Pickard, C. J.; Hasnip, P. J.; Clark, S. J.; Payne, M. C. First-Principles Simulation: Ideas, Illustrations and the CASTEP Code. $J$. Phys. Condens. Matter. 2002, 14, 2717-2744.

[3] Perdew, J. P.; Burke, K.; Ernzerhof, M. Generalized Gradient Approximation Made Simple. Phys. Rev. Lett. 1996, 77, 3865.

[4] Lin, J. S.; Qteish, A.; Payne, M. C.; Heine, V. V.; Optimized and transferable nonlocal separable ab initio pseudopotentials. Phys. Rev. B: Condens. Matter Mater. Phys. 1993, $47,4174$.

[5] Cabellos, J. L.; Mendoza, B. S.; Escobar, M. A.; Nastos, F.; Sipe, J. E. Effects of nonlocality on second-harmonic generation in bulk semiconductors. Phys. Rev. B: Condens. Matter. 2009, 80, 155205.

[6] Lo, C. H.; Lee, M. H.; Master Thesis, Taiwan, 2005. 